\title{
Search for neutralino dark matter with NaI detectors
}

\author{
A. Bottino, V. de Alfaro, N. Fornengo, G. Mignola, S. Scopel \\ Dipartimento di Fisica Teorica dell'Università di Torino and INFN, Sezione di Torino, Turin, Italy
}

Beijing - Roma - Saclay (BRS) Collaboration

C. Bacci ${ }^{a}$, P. Belli ${ }^{b}$, R. Bernabei ${ }^{b}$, Dai Changjiang ${ }^{c}$, Ding Linkai ${ }^{c}$, E. Gaillard ${ }^{d}$, G. Gerbier $^{\text {d }}$, Kuang Haohuai ${ }^{\text {c}}$, A. Incicchitti a , J. Mallet ${ }^{\text {d, R. Marcovaldi }}{ }^{\text {a }}$, L. Mosca ${ }^{\text {d, D. Prosperi }}{ }^{\text {a }}$, C. Tao ${ }^{1, \mathfrak{c}, \mathrm{d}}$ and Xie Yigang ${ }^{\mathrm{c}}$

" Dipartimento di Fisica, Università di Roma "La Sapienza" and INFN, Sezione di Roma, I-00185 Rome, Italy

- Dipartimento di Fisica, Università di Roma "Tor Vergata" and INFN, Sezione di Roma 2, I-00173 Rome, Italy

c Beijing, China (IHEP), Academia Sinica, P.O. Box 918/3, Beijing, China

d DSMIDAPNIA/SPP, C.E. Saclay, F-91191 Gif-sur-Yvette, France

Received 23 September 1992

\begin{abstract}
Theoretical predictions for neutralino dark matter in the framework of the minimal SUSY standard model are compared with recent results of a direct search for dark matter with NaI detectors. Perspectives for a future investigation by this kind of detectors are also discussed.
\end{abstract}

\section{Introduction}

An experimental for direct detection of weakly interacting massive particles (WIMPs) using NaI crystals is in progress and preliminary results have been reported [1]. In the present paper we compare the experimental data of ref. [1] with the theoretical expectations based on one of the favourite particle candidates for cold dark matter: the neutralino. In the light of the theoretical predictions we discuss the perspectives of future experimental searches of neutralino dark matter employing $\mathrm{NaI}$ detectors.

For the general theoretical aspects of the neutralino as dark matter candidate and for other (indirect) ways of searching for it we refer to refs. [2-4]. The simplest version of the SUSY theories is the minimal supersymmetric standard model (MSSM), appropriately implemented by some GUT assumptions. This constitutes the theoretical framework employed in the present paper.

\footnotetext{
1 Also at LPC, Collège de France, IN2P3/CNRS, France.
}

For the direct search of the WIMPs the most useful quantity is the nuclear recoil spectrum $[5,6]$

$$
\frac{\mathrm{d} R}{\mathrm{~d} E_{\mathrm{R}}}=N_{\mathrm{T}} \frac{\rho_{\chi}}{m_{\chi}} \int_{v_{\min }\left(E_{\mathrm{R}}\right)}^{\nu_{\max }} \mathrm{d} v f(v) v \frac{\mathrm{d} \sigma}{\mathrm{d} E_{\mathrm{R}}}\left(v, E_{\mathrm{R}}\right) .
$$

$N_{\text {T }}$ is the number density of the detector nuclei, $\rho_{x} /$ $m_{\chi}$ is the local number density of the dark matter particles (here neutralinos, denoted by $\chi$ ) and $f(v)$ is their galactic velocity distribution in the Earth rest frame, $\mathrm{d} \sigma / \mathrm{d} E_{\mathrm{R}}$ is the differential neutralino-nucleus cross section, where $E_{\mathrm{R}}$ is the nucleus recoil energy $E_{\mathrm{R}}=m_{\mathrm{red}}^{2} v^{2}\left(1-\cos \theta^{*}\right) / m_{\mathrm{N}}$. Here $\theta^{*}$ denotes the scattering angle in the neutralino-nucleus center of mass frame; $m_{N}$ is the nucleus mass and $m_{\text {red }}$ the neutralino-nucleus reduced mass.

The velocity $v_{\min }\left(E_{\mathrm{R}}\right)$ is given by $v_{\min }\left(E_{\mathrm{R}}\right)=$ $\left(m_{\mathrm{N}} E_{\mathrm{R}} / 2 m_{\text {red }}^{2}\right)^{1 / 2}$ and $v_{\max }$ is the maximal neutralino velocity in the halo (i.e. the galaxy escape velocity) computed in the Earth reference frame.

In the following we use for $\mathrm{d} \sigma\left(v, E_{\mathrm{R}}\right) / \mathrm{d} E_{\mathrm{R}}$ its low energy approximation, i.e. 
$\frac{\mathrm{d} \sigma}{\mathrm{d} E_{\mathrm{R}}}\left(v, E_{\mathrm{R}}\right)=\frac{\mathrm{d} \sigma}{\mathrm{d} E_{\mathrm{R}}}(v, 0) F^{2}\left(E_{\mathrm{R}}\right)$,

where $F\left(E_{\mathrm{R}}\right)$ denotes a form factor due to the finite size of the nucleus, and $\left(\mathrm{d} \sigma / \mathrm{d} E_{\mathrm{R}}\right)(v, 0)$ may be conveniently expressed as

$\frac{\mathrm{d} \sigma}{\mathrm{d} E_{\mathrm{R}}}(v, 0)=\frac{\sigma}{E_{\mathrm{R}}^{\max }}$.

Here $E_{\mathrm{R}}^{\max }=2 m_{\mathrm{red}}^{2} v^{2} / m_{\mathrm{N}}$ and $\sigma$ is the total elastic cross section computed for a pointlike nucleus and for zero threshold recoil energy. Then eq. (1) can be rewritten as

$\frac{\mathrm{d} R}{\mathrm{~d} E_{\mathrm{R}}}=\frac{R_{0}}{\left\langle E_{\mathrm{R}}^{\max }\right\rangle} F^{2}\left(E_{\mathrm{R}}\right) I\left(E_{\mathrm{R}}\right)$,

where $\left\langle E_{\mathrm{R}}^{\max }\right\rangle$ is the average of $E_{\mathrm{R}}^{\max }$ over the velocity distribution, $R_{0}$ is a total rate defined as

$R_{0}=N_{\mathrm{T}} \frac{\rho_{x}}{m_{x}} \sigma\langle v\rangle$,

and

$I\left(E_{\mathrm{R}}\right)=\frac{\left\langle v^{2}\right\rangle}{\langle v\rangle} \int_{v_{\min }\left(E_{\mathrm{R}}\right)}^{\nu_{\max }} \mathrm{d} v \frac{f(v)}{v}$.

Taking for the $\chi$ 's a maxwellian velocity distribution (in the galactic rest frame) of root mean square velocity $w$ and putting $v_{\max }=\infty, I\left(E_{\mathrm{R}}\right)$ becomes

$$
\begin{aligned}
& I\left(E_{\mathrm{R}}\right)=\frac{\sqrt{\pi}}{2} \frac{3+2 \eta^{2}}{\sqrt{\pi}\left(1+2 \eta^{2}\right) \operatorname{erf}(\eta)+2 \eta \exp \left(-\eta^{2}\right)} \\
& \quad \times\left[\operatorname{erf}\left(x_{\min }+\eta\right)-\operatorname{erf}\left(x_{\min }-\eta\right)\right],
\end{aligned}
$$

where

$$
\begin{gathered}
\eta=\sqrt{\frac{3 v_{\text {Earth }}^{2}}{2 w^{2}}}, \quad x_{\min }=\sqrt{\frac{3 m_{\mathrm{N}} E_{\mathrm{R}}}{4 m_{\mathrm{red}}^{2} w^{2}}}, \\
\operatorname{erf}(x)=\frac{2}{\sqrt{\pi}} \int_{0}^{x} \exp \left(-t^{2}\right) \mathrm{d} t ;
\end{gathered}
$$

here $v_{\text {Earth }}$ denotes the Earth velocity in the galactic frame. In our calculations we take $\left|\boldsymbol{v}_{\text {Earth }}\right|=245$ $\mathrm{km} \mathrm{s}^{-1}$ (here the effect of the flux annual modulation due to the Earth-Sun relative motion is neglected), $w=300 \mathrm{~km} \mathrm{~s}^{-1}$ which implies $\langle v\rangle=361 \mathrm{~km} \mathrm{~s}^{-1}$. We notice that all the averages over the velocity distri- bution introduced here are meant to be evaluated in the Earth rest frame.

To obtain the recoil spectrum in the case of neutralino DM, in eqs. (4), (5) we need to evaluate the quantities $\rho_{\chi}$ and $\sigma$ in an appropriate SUSY model.

\section{Theoretical framework}

As mentioned above, our framework is the MSSM. The neutralino is defined to be the lowest-mass linear superposition of photino, zino and higgsinos,

$\chi=a_{1} \tilde{\gamma}+a_{2} \tilde{Z}+a_{3} \tilde{H}_{1}^{0}+a_{4} \tilde{H}_{2}^{0}$.

We adopt the usual definitions of $\tilde{\gamma}$ and $\tilde{Z}$ in terms of the neutral $\mathrm{U}(1), \mathrm{SU}(2)$ gauginos $\left(\tilde{B}\right.$ and $\tilde{W}_{3}$, respectively):

$\tilde{\gamma}=\cos \theta_{\mathrm{w}} \tilde{B}+\sin \theta_{\mathrm{w}} \tilde{W}_{3}$,

$\tilde{Z}=-\sin \theta_{\mathrm{w}} \tilde{B}+\cos \theta_{\mathrm{w}} \tilde{W}_{3}$,

where $\theta_{\mathrm{w}}$ is the Weinberg angle.

In the MSSM the coefficients $a_{i}$ and the neutralino mass are functions of a restricted number of parameters, which may be chosen to be the $\widetilde{B}$ mass parameter $M_{1}$, the $\tilde{W}_{3}$ mass parameter $M_{2}$, the Higgs mixing mass parameter $\mu$ and the ratio of the VEVs of the two Higgs doublets $v_{2} / \nu_{1}=\tan \beta$ ( $v_{1}$ and $v_{2}$ are the VEVs of the doublets which give masses to the downtype quarks and to the up-type quarks, respectively). The standard GUT condition $M_{1}=\frac{5}{3} M_{2} \tan ^{2} \theta_{\mathrm{w}}$ is assumed to hold. Further parameters are the masses of the two neutral $C P$-even Higgs bosons $(h, H)$ and of the $C P$-odd one $(A)$. If one of the Higgs masses, say $m_{h}$, is taken to be a free parameter, the other two masses $m_{A}, m_{H}$ may be expressed in terms of $m_{h}$, of the mass of the top quark $m_{t}$ and of the mass $\tilde{m}$ of its SUSY partners (taken to be degenerate). This last property relies on recent results about one-loop radiative corrections to the Higgs masses [7].

As for the values of the $\chi$ mass, $m_{\chi}$, the range considered in this paper is $20 \mathrm{GeV} \leqslant m_{\chi} \leqslant m_{W}$, which is above the present experimental lower bound [8] ${ }^{\# 1}$,

\#1 It has recently been shown [9] that a model of non-linear evolution of initial density fluctuations in the universe, under the further assumption that the average $\chi$ density in the universe is $70 \%$ of the critical density, points to lower limits on $m_{x}$ much larger than those obtained at accelerators. 
but within the reach of the next future accelerators. Extension of the present analysis to neutralinos heavier than the $W$ boson is under way.

In the MSSM the total cross section $\sigma$ can be evaluated as described in refs. [2-4]. The relevant amplitudes are due to Higgs boson exchange and $Z_{0}$ exchange in the $t$-channel and to squark exchange in the $s$-channel. The crucial properties are the following: (1) Higgs boson exchange contributes a coherent $(\mathrm{CH})$ cross section which is non-zero only for a $\chi$ whose composition contains a zino-higgsino mixture [10]; (2) $Z_{0}$ exchange provides a spin-dependent (SD) cross section which takes contribution only from the higgsino components of $\chi$; (3) squark exchange contributes a $\mathrm{CH}$ cross section (due to zinohiggsino mixing) as well as a SD cross section (mainly due to the gaugino components in $\chi$ ) [11]. Because of the relative size of the various couplings and of the fact that the squark mass is already severely constrained by present accelerator data $(\tilde{m}>150 \mathrm{GeV})$, in what follows we disregard the squark-exchange diagrams (see below for a further comment on this point).

Under these assumptions the coherent cross section is [10]

$\sigma_{\mathrm{CH}}=\frac{8 G_{\mathrm{F}}^{2}}{\pi} \frac{m_{Z}^{2}}{m_{h}^{4}} \alpha_{\mathrm{H}}^{2} m_{\mathrm{red}}^{2} A^{2}$,

where $A$ is the mass number of the nucleus and $\alpha_{\mathrm{H}}$ is an effective coupling constant which depends on the MSSM parameters (the explicit expression for $\alpha_{\mathrm{H}}$ and its numerical values are given in ref. [3] ).

The SD cross section due to $Z_{0}$ exchange is given by $[11,12]$

$\sigma_{\mathrm{SD}}=\frac{8 G_{\mathrm{F}}^{2}}{\pi}\left(a_{3}^{2}-a_{4}^{2}\right)^{2} m_{\mathrm{red}}^{2}\left(\Sigma_{q} T_{3 \mathrm{~L}, q} \Delta q\right)^{2} \lambda^{2} J(J+1)$.

The values of $\lambda^{2}$ used in this paper are those obtained in the odd-group model $[13,14]$ where only the odd nuclear species in odd-even nuclei is explicitly taken into account. Thus in eq. (11) $\Delta q$ is the fractional spin carried by the quark $q$ in the nucleon of the odd species; $T_{3 \mathrm{~L}, q}$ denotes the third component of the quark weak isospin. Other determinations of $\lambda^{2}$ are given in ref. [15].

In ref. [2] $\sigma$ has been evaluated in extended regions of the parameters space for a number of repre- sentative points defined by fixed values of the following parameters: $m_{h}, \tan \beta, m_{t}$ and $\tilde{m}$. For each representative point the other free parameters, $M_{2}$ and $\mu$, have been varied in the ranges $0<M_{2}<600 \mathrm{GeV}$, $-300<\mu<300 \mathrm{GeV}$.

The choice of the representative points in ref. [2] was meant to illustrate different physical scenarios of particular significance. Here we consider the following values: $\tan \beta=2$ and $8, m_{t}=150 \mathrm{GeV}, \tilde{m}=1 \mathrm{TeV}$, $m_{h} \gtrsim 50 \mathrm{GeV}$ (see LEP lower bounds for SUSY Higgs bosons [8]).

\section{Cross sections and rates for $\mathrm{NaI}$ target-detectors}

To analyze the preliminary results from the $\mathrm{NaI}$ experiment mentioned in the introduction we are now focusing on the sodium and iodine target-nuclei. Experimental details can be found in ref. [1], from which we quote some parameter values relevant to this analysis. The present value of the electron-equivalent energy threshold $E_{\mathrm{ee}}^{\text {th }}$ is about $4 \mathrm{keV}$. The scintillation efficiency is lower for a recoiling nucleus than for an electron of the same kinetic energy: the corresponding ratio, or "quenching factor", is $\simeq 0.25$ for a ${ }^{23} \mathrm{Na}$ and $\simeq 0.07$ for a ${ }^{127} \mathrm{I}$ nucleus, so the recoil energy threshold is $E_{\mathrm{R}}^{\text {th }} \simeq 16 \mathrm{keV}$ for sodium and $E_{\mathrm{R}}^{\text {th }} \simeq 57 \mathrm{keV}$ for iodine. At these energies the present counting rate is about 10 events $/ \mathrm{kg} / \mathrm{keV} / \mathrm{d}$.

\subsection{Coherent pointlike cross section}

This is maximal in the parameter-space regions where the zino-higgsino mixing is dominant, i.e. in the $\mu>0$ sector. Here, at $\tan \beta=8, \alpha_{\mathrm{H}}$ turns out to be in the range $\alpha_{\mathrm{H}}=1.2-1.5 \mathrm{GeV}$; this implies, from eq. (10),

$\sigma_{\mathrm{CH}}=(2-8) \mathrm{nb}\left(\frac{50 \mathrm{GeV}}{m_{h}}\right)^{4}$ for ${ }^{127} \mathrm{I}$,

and

$\sigma_{\mathrm{CH}}=(2-3) \times 10^{-2} \mathrm{nb}\left(\frac{50 \mathrm{GeV}}{m_{h}}\right)^{4}$ for ${ }^{23} \mathrm{Na}$.

For this $\tan \beta$ value, in the sector $\mu<0$ the cross sections are only reduced by a factor $1.5-2.0$ as com- 
pared to the values given in eqs. $\left(10^{\prime}\right),\left(10^{\prime \prime}\right)$. However, at lower values of $\tan \beta$, the effective coupling constant $\alpha_{\mathrm{H}}$ suppresses the coherent cross sections significantly. For instance, at $\tan \beta=2$ the $\sigma_{\mathrm{CH}}$ values in the $\mu>0(\mu<0)$ sector are drown by one (two) orders of magnitude with respect to the cross-sections values reported in eqs. $\left(10^{\prime}\right),\left(10^{\prime \prime}\right)$.

\subsection{Spin-dependent pointlike cross sections}

Eq. (11), computed with the numerical values [14] $\lambda^{2} J(J+1)=0.041$ for ${ }^{23} \mathrm{Na}, \lambda^{2} J(J+1)=0.007$ for ${ }^{127} \mathrm{I} ; \Delta u=0.77, \Delta d=-0.49, \Delta s=-0.15$ and our evaluations of the coefficients $a_{3}, a_{4}$, leads to the following estimates for the maximal contributions due to the SD effects:

$\sigma_{\mathrm{SD}} \lesssim 1 \times 10^{-4} \mathrm{nb}$ for ${ }^{127} \mathrm{I}$

and

$\sigma_{\mathrm{SD}} \lesssim 2 \times 10^{-4} \mathrm{nb}$ for ${ }^{23} \mathrm{Na}$.

Possible SD contributions due to $\tilde{q}$-exchange would provide cross sections of a few $10^{-4}\left(m_{W} / m_{\tilde{q}}\right)^{4} \mathrm{nb}$ in regions where $\chi$ is dominantly a gaugino.

From the previous discussion it follows that, for scattering of $\chi$ off point-like ${ }^{23} \mathrm{Na}$ and ${ }^{127} \mathrm{I}$ nuclei, $\mathrm{CH}$ cross sections largely dominate over the SD cross sections, except where coherent effects are severely hindered by small values of $\tan \beta$ and by values of $m_{h}$ which are well above the present experimental bound. This happens, for example, at $\tan \beta=2, m_{h}=80 \mathrm{GeV}$; however, here the level of the total cross sections is very low (about $8 \times 10^{-5} \mathrm{nb}$ ).

\subsection{Nuclear form factors}

The specific analytic expressions to be used for the form factors depend on the associated elastic nuclear cross section. In the case of coherent cross section it may be taken of the form [18]

$F\left(E_{\mathrm{R}}\right)=\frac{3 j_{1}\left(q r_{0}\right)}{q r_{0}} \exp \left(-\frac{1}{2} s^{2} q^{2}\right)$.

Here $q^{2} \equiv|q|^{2}=2 m_{N} E_{\mathrm{R}}$ is the squared three-momentum transfer, $s \simeq 1 \mathrm{fm}$ is the thickness parameter for the nucleus surface, $r_{0}=\left(r^{2}-5 s^{2}\right)^{1 / 2}, r=1.2 \mathrm{~A}^{1 / 3}$ fm and $j_{1}\left(q r_{0}\right)$ is the spherical Bessel function of index 1 .
For heavy nuclei the suppression effect introduced by the form factor in the differential rate is noticeable at threshold already. In fact, using the values of $E_{\mathrm{ee}}^{\text {th }}$ and of the quenching factors given above one finds from eq. (12) that $F^{2}\left(E_{\mathrm{R}}^{\text {th }}\right)=0.96$ for ${ }^{23} \mathrm{Na}$ and $F^{2}\left(E_{\mathrm{R}}^{\text {th }}\right)=0.045$ for ${ }^{127} \mathrm{I}$. For ${ }^{23} \mathrm{Na}$ the differential rate is negligibly affected by the form factor over the whole $E_{\mathrm{R}}$ spectrum. On the contrary, for ${ }^{127} I$ the form factor plays an important role in reducing the coherent cross section also because of the first zero of the function $j_{1}\left(q r_{0}\right) / q r_{0}$ which occurs at a rather low value of $E_{\text {ee }}$, i.e. at $E_{\mathrm{ee}} \simeq 7.3 \mathrm{keV}$.

For the SD cross section the relevant form factor has a smaller suppression effect in heavy nuclei, as was shown in ref. [16] for the case of ${ }^{131} \mathrm{Xe}$ and in ref. [17] for ${ }^{93} \mathrm{Nb}$. A corresponding nuclear evaluation for ${ }^{127} \mathrm{I}$ is not available at present ${ }^{\# 2}$; so in this paper we have simply assumed that the form factor of the SD amplitude in ${ }^{127} \mathrm{I}$ and in ${ }^{131} \mathrm{Xe}$ are roughly the same and we have used the results of ref. [16].

\subsection{Neutralino local density}

We may take for it the standard value agreed for the local DM density, i.e. $\rho_{\ell}=0.2-0.4 \mathrm{GeV} / \mathrm{cm}^{3}$, whenever the value of the average relic density, $\Omega_{x} h^{2}$, is $\gtrsim 0.05$. When $\Omega_{\chi} h^{2}$ is smaller than this value because the $\chi \chi$ annihilation cross section, $\sigma_{\text {ann }}$, is large (and so the neutralino cannot be the only component of DM), $\rho_{\chi}$ has to be rescaled [19,20,2]; thus we take $\rho_{\chi}=\xi \rho_{\ell}$, with $\xi=\Omega_{x} h^{2} / 0.05$ whenever $\Omega_{x} h^{2}<0.05$, $\xi=1$ otherwise. It is worth remarking that $\Omega_{\chi} h^{2}$ is strongly suppressed around the point $m_{x}=\frac{1}{2} m_{A}$ because at this value of $m_{\chi}$ the Higgs-exchange amplitude in $\sigma_{\text {ann }}$ has a pole singularity. The values of $\Omega_{\chi} h^{2}$ used here for rescaling are taken from ref. [2] and refer to the same parameter space discussed above in connection with the cross sections.

\subsection{Results}

Our results are reported in figs. 1-3. Fig. 1 shows the maximal differential rate, $\mathrm{d} R / \mathrm{d} E_{\mathrm{ee}}$, which was found for $\mathrm{NaI}$ by varying $M_{2}$ and $\mu$ in the ranges mentioned above, at the representative point defined by $\tan \beta=8, m_{h}=50 \mathrm{GeV}$; here the $\chi$ local density is set

\footnotetext{
\#2 A theoretical investigation on this subject is in progress.
} 


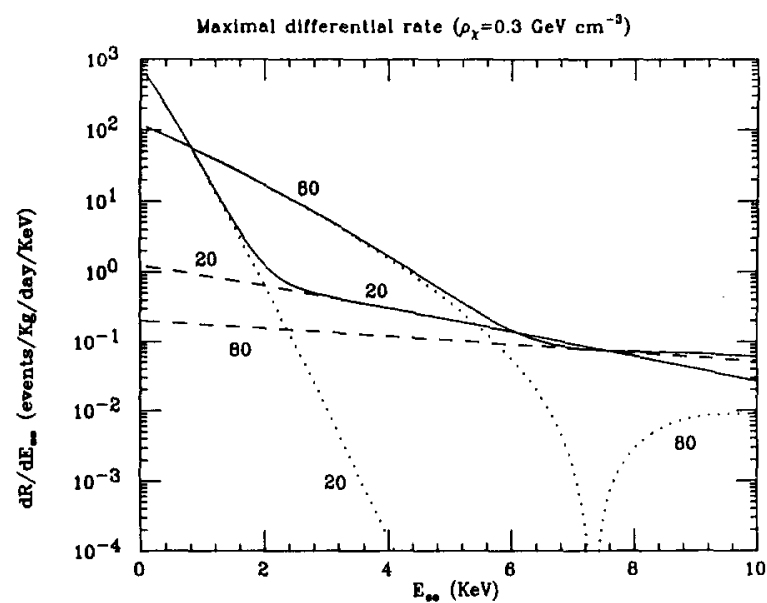

Fig. 1. Maximal differential rate, $\mathrm{d} R / \mathrm{d} E_{\text {ee }}$, at $\tan \beta=8, m_{h}=50$ $\mathrm{GeV}, m_{t}=150 \mathrm{GeV}, \tilde{m}=1 \mathrm{TeV}$ when $M_{2}$ and $\mu$ are varied in the range: $0<M_{2}<600 \mathrm{GeV},-300<\mu<300 \mathrm{GeV}$. Here $\rho_{x}$ is set equal to the local DM density: $\rho_{x}=\rho_{\ell}=0.3 \mathrm{GeV} \mathrm{cm}^{-3}$. Dotted, dashed and solid lines refer to ${ }^{127} \mathrm{I},{ }^{23} \mathrm{Na}$ and total $\mathrm{Nal}$ contributions, respectively, at two values of the $\chi$ mass: $m_{\chi}=20,80 \mathrm{GeV}$.

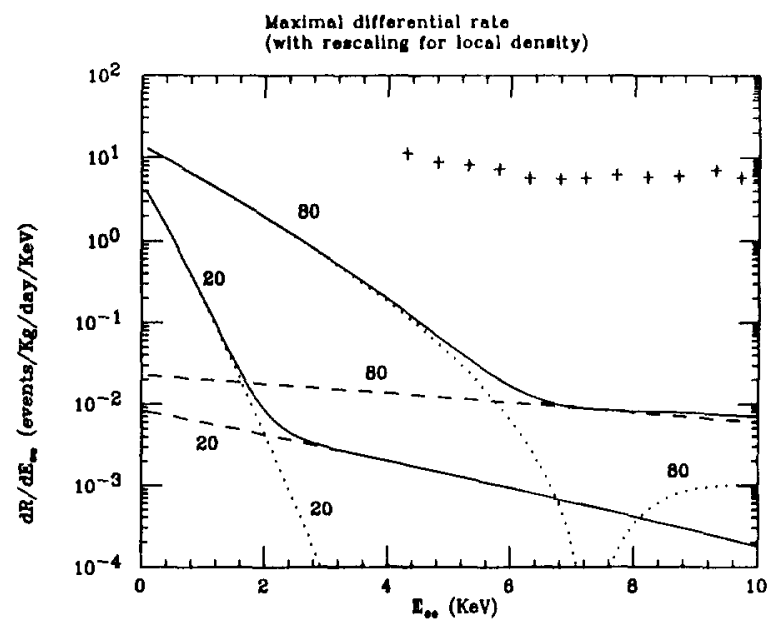

Fig. 2. The same as in fig. 1, except that here $\rho_{\chi}$ is rescaled $\left(\rho_{\chi}=\xi \rho_{\ell}\right)$ according to the procedure discussed in the text. The crosses denote the experimental upper limits of ref. [1].

equal to the standard value for the local DM density: $\rho_{\chi}=\rho_{\ell}=0.3 \mathrm{GeV} \mathrm{cm}{ }^{-3}$. The dotted line which denotes the contribution of iodine for $m_{x}=80 \mathrm{GeV}$ shows a typical dip at $E_{\mathrm{ee}} \simeq 7.3 \mathrm{keV}$, due to a zero of the form factor in eq. (12). In fig. 2 we report the maximal rescaled differential rate $\mathrm{d} R / \mathrm{D} E_{\text {ee }}$, calculated in the same representative point as in fig. 1 . As

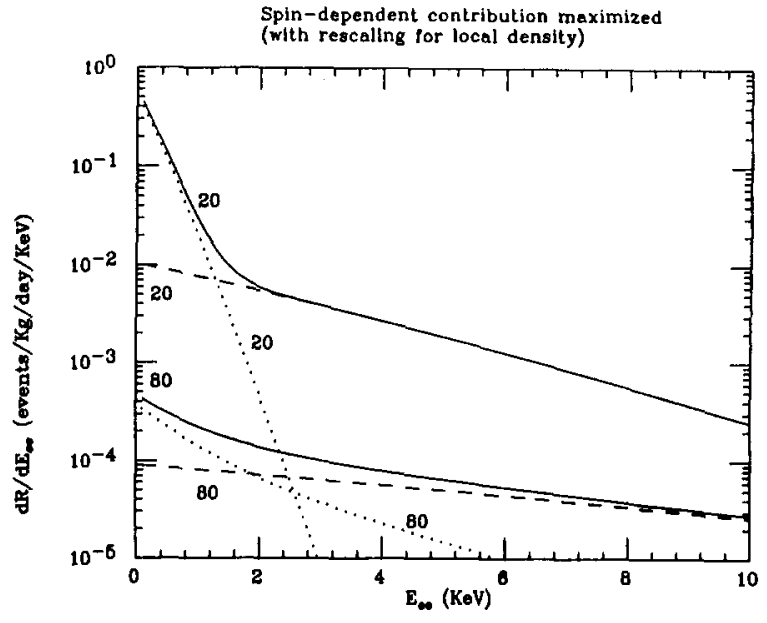

Fig. 3. Differential rate when the SD contribution is maximized by varying $M_{2}, \mu$ in the same range as in figs. 1,2 . The representative point is defined here by $\tan \beta=2, m_{h}=80 \mathrm{GeV}, m_{t}=150$ $\mathrm{GeV}, \tilde{m}=1 \mathrm{TeV}$. The conventions for the symbols are as in the previous figures.

previously discussed, rescaling is introduced in $\mathrm{d} R$ / $\mathrm{d} E_{\text {ee }}$ by putting $\rho_{\chi}=\xi_{\ell}$ in eq. (5). Here $\mathrm{d} R / \mathrm{d} E_{\mathrm{ee}}$ is dominated by coherent effects over the whole shown range (the ratio of the $\mathrm{SD}$ contribution to the $\mathrm{CH}$ contribution is typically $\left.\mathrm{O}\left(10^{-3}\right)\right)$. Here the rescaling suppresses the $m_{x}=20 \mathrm{GeV}$ curves in a very substantial way $\left(\xi \simeq 8 \times 10^{-3}\right)$, because the pole of $\sigma_{\mathrm{ann}}$ occurs at $m_{\chi} \simeq 25 \mathrm{GeV}$ (in fact at the present representative point the value of $m_{A}$ turns out to be $m_{A}=51$ $\mathrm{GeV}$ ). The differential rate for values of $m_{\chi}$ in the range $m_{\chi}=40-80 \mathrm{GeV}$ (the $m_{\chi}=80 \mathrm{GeV}$ case is shown in fig. 2 ) is rescaled much less, i.e. $\xi \simeq 0.2-0.4$. In fig. 2 the crosses denote the present experimental upper limits of ref. [1].

We clearly see from fig. 2 the interplay between the two nuclei, ${ }^{23} \mathrm{Na}$ and ${ }^{127} \mathrm{I}$, in determining the total differential rate. For instance, for $m_{x}=80 \mathrm{GeV}$, up to the value $E_{\mathrm{ee}} \simeq 6 \mathrm{keV}, \mathrm{d} R / \mathrm{d} E_{\mathrm{ee}}$ is dominated by scattering of $\chi$ off iodine.

One may wonder whether SD effects could provide a sizeable differential rate for a $\chi$ of some appropriate composition (e.g. a $\chi$ with higgsino dominance in the case of a $Z$-exchange graph). Our previous estimates for the relevant cross sections, eqs. (11), show that the SD effects are in general very small in the parameter space considered in this paper; a direct numerical test of maximization of the SD contributions (in- 
stead of the total, coherent plus SD, differential rate) confirms these expectations.

The results obtained by applying this maximization procedure to the rescaled $\mathrm{d} R / \mathrm{d} E_{\text {ee }}$ distribution are shown in fig. 3. Apart from the low values of $m_{x}$ ( $m_{\chi} \simeq 20 \mathrm{GeV}$ ), the SD contribution is maximal and dominant over the $\mathrm{CH}$ contribution in the parameter region where $\mu<0$. The overall signal shown in fig. 3 remains rather low in the whole $m_{\chi}$ range.

From the point of view of the rescaling effect we remark that at the representative point of fig. 3 $\left(\tan \beta=2, m_{h}=80 \mathrm{GeV}\right)$ the value of $m_{A}$ is $m_{A}=170$ $\mathrm{GeV}$ and then rescaling is particularly severe for $m_{\chi} \simeq 80 \mathrm{GeV}$. The $\mathrm{d} R / \mathrm{d} E_{\text {ee }}$ distributions is much less affected by the rescaling procedure for values of $m_{x}$ in the ranges $m_{x}=20-30 \mathrm{GeV}$ (the case $m_{x}=20 \mathrm{GeV}$ is shown in fig. 3 ) and $m_{\chi}=50-60 \mathrm{GeV}$. At $m_{\chi} \simeq 40$ $\mathrm{GeV}$ a dip occurs in $\Omega_{\chi} h^{2}$ because of a singularity due to $h$-exchange in the annihilation amplitude [2].

\section{Conclusions and outlook}

In the present paper theoretical predictions based on MSSM are compared to preliminary data from an $\mathrm{NaI}$ scintillator experiment. The total elastic $\chi-\mathrm{NaI}$ differential rate is maximal in the MSSM parameters space region where the coherent $\chi$-nucleus cross section is dominant due to zino-higgsino mixing. In this case the experimental sensitivity has to be improved by about two-three orders of magnitude in order to start testing the predictions of the model in the $\chi$-mass region around $40-80 \mathrm{GeV}$. Quantitatively the situation is given in fig. 2. It must be emphasized that the ${ }^{127}$ I contribution, dominant over the ${ }^{23} \mathrm{Na}$ contribution for $m_{\chi} \approx 80 \mathrm{GeV}$, directly depends on the value of the iodine quenching factor which still needs to be determined accurately.

If the zino-higgsino mixing in the neutralino composition is very small, the coherent cross sections are strongly reduced. In this case the ratio of the spin dependent contribution to the coherent contribution may be substantial (in particular for ${ }^{23} \mathrm{Na}$ ); however the relevant differential rate is one or two orders of magnitude lower than the values taken by the coherent rate in the regions where this last one is maximal.

From the experimental point of view an increase of sensitivity of two orders of magnitude may be reached in the next few years by a better selection and purification of all the detector components, an improvement of the shielding thickness and quality, a possible statistical separation between Compton electrons versus nuclear recoils, and a study of the WIMPs flux annual modulation.

Such an improvement will allow to start testing the present model. It also provides the opportunity to explore a region of masses and cross sections which has never been reached so far, where other presently unpredicted DM candidates could be present.

Moreover other scintillation crystals, in principle more favourable than sodium and iodine for the spin dependent and/or coherent couplings (like $\mathrm{CaF}_{2}$ ), are under investigation in the BRS Collaboration.

\section{References}

[1] Beijing - Roma - Saclay (BRS) Collab., C. Bacci et al., LNGS preprint $92 / 36$.

[2] A. Bottino, V. de Alfaro, N. Fornengo, G. Mignola and S. Scopel, University of Torino preprint DFTT 33/92, Astropart. Phys. 1 (1992), to appear;

A. Bottino, V. de Alfaro, N. Fornengo, G. Mignola and M. Pignone, Phys. Lett. B 265 (1991) 57, and references therein.

[3] A. Bottino, V. de Alfaro, N. Fornengo, A. Morales, J. Puimedón and S. Scopel, Mod. Phys. Lett. A 7 (1992) 733; in: TAUP91 Proc., eds. A. Morales, J. Morales and J.A. Villar, Nucl. Phys. B (Proc. Suppl.) 28 A (1992) 314.

[4] P. Fayet, Phys. Lett. B 86 ( 1979 ) 272;

R. Flores, K.A. Olive and M. Srednicki, Phys. Lett. B 237 (1990) 72;

J. Ellis, L. Roszkowski and Z. Lalak, Phys. Lett. B 245 (1990) 545 ;

J.L. Lopez, D.V. Nanopoulos and Kajia Yuan, preprint CTPTAMU-31/91 (1991);

L. Roszkowski, Phys. Lett. B 262 ( 1991 ) 59;

K. Griest and L. Roskowski, preprint CERN-TH 6181/91; J. McDonald, K.A. Olive and M. Srednicki, preprint UMNTH 1017/92;

J. Ellis and L. Roskowski preprint CERN-TH 6260/91; V. Berezinsky, invited talk at Neutrino 92 (Granada, June 1992), Nucl. Phys. Suppl. (to appear), and references therein.

[5] A.K. Drukier, K. Freese and D.N. Spergel, Phys. Rev. D 33 (1986) 3495;

A. Gould, Astrophys. J. 321 (1987) 571;

K. Freese, J. Frieman and A. Gould, Phys. Rev. D 37 ( 1988) 3388.

[6] J.R. Primack, B. Sadoulet and D. Seckel, Annu. Rev. Nucl. Sci. 38 ( 1988 ) 751 ;

P.F. Smith and J.D. Lewin, Phys. Rep. 187 (1990) 203. 
[7] Y. Okada, M. Yamaguchi and T. Yanagida, preprint YU360 (1990);

J. Ellis, G. Ridolfi and F. Zwirner, Phys. Lett. B 257 (1991) 83;

H. Haber and R. Hempfling, Phys. Rev. Lett. 66 (1991) 1815

R. Barbieri, M. Frigeni and F. Caravaglios, Phys. Lett. B 258 (1991) 167;

R. Barbieri and R. Frigeni, Phys. Lett. B 258 (1991) 395;

J.L. Lopez and D.V. Nanopoulos, Phys. Lett. B 266 (1991) 397;

A. Brignole, J. Ellis, G. Ridolfi and F. Zwirner, Phys. Lett. B 271 (1991) 123;

V. Barger, M.S. Berger, A.L. Stange and R.J.N. Phillips, preprint MAD/PH/680 (November 1991);

A. Brignole, preprint CERN-TH-6366/92 (January 1992).

[8] ALEPH Collab., D. Decamp et al., Phys. Rep. 216 (1992) 253;

L. Roszkowski, Phys. Lett. B 262 ( 1991 ) 59;

K. Hidaka, Phys. Rev. D 44 (1991) 927.

[9] V.S. Berezinsky, A.V. Gurevich and K.P. Zybin, LNGS preprint $92 / 40$.
[10] R. Barbieri, M. Frigeni and G.F. Guidice, Nucl. Phys. B 313 (1989) 725.

[11] K. Griest, Phys. Rev. D 38 (1988) 2357; Phys. Rev. Lett. 61 (1988) 666.

[12] M.K. Goodman and E. Witten, Phys. Rev. D 31 (1985) 3059.

[13] J. Engel and P. Vogel, Phys. Rev. D 40 (1989) 3132.

[14] J. Ellis and R. Flores, Phys. Lett. B 263 (1991) 259; preprint CERN-TH-6483/92.

[15] A.F. Pacheco and D.D. Strottman, Phys. Rev. D 40 (1989) 2131;

F. Iachello, L.M. Krauss and G. Maino, Phys. Lett. B 254 (1991) 220.

[16] J. Engel, Phys. Lett. B 264 (1991) 114.

[17] J. Engel, S. Pittel, E. Ormand and P. Vogel, Phys. Lett. B 275 (1992) 119.

[18] Q. Chengrui and H. Tsehsui, to appear.

[19] T.K. Gaisser, G. Steigman and S. Tilav, Phys. Rev. D 34 (1986) 2206.

[20] G.B. Gelmini, P. Gondolo and E. Roulet, Nucl. Phys. B 351 (1991) 623. 